\title{
A REMARKABLE ANGULAR DISTRIBUTION OF THE INTERMEDIATE SUBCLASS OF GAMMA-RAY BURSTS
}

\author{
Attila MészÁros, ${ }^{1,2,3}$ Zsolt Bagoly, ${ }^{4}$ István Horváth, ${ }^{5}$ Lajos G. Balázs, ${ }^{2}$ AND Roland VavreK ${ }^{2,6}$ \\ Received 1999 July 21 ; accepted 2000 March 16
}

\begin{abstract}
We develop a method of testing the null hypothesis of intrinsic randomness in the angular distribution of gamma-ray bursts collected in the Current BATSE Catalog. The method is a modified version of the well-known counts-in-cells test and fully eliminates the nonuniform sky-exposure function of the BATSE instrument. Applying this method to the case of all gamma-ray bursts, we found no intrinsic nonrandomness. The test also did not find intrinsic nonrandomness for the short and long gamma-ray bursts. However, using the method on the new, intermediate subclass of gamma-ray bursts, the null hypothesis of intrinsic randomness for 181 intermediate gamma-ray bursts is rejected on the $96.4 \%$ confidence level. Taking 92 dimmer bursts from this subclass, we obtain a surprising result: this "dim" subclass of the intermediate subclass has an intrinsic nonrandomness on the $99.3 \%$ confidence level. On the other hand, the 89 "bright" gamma-ray bursts show no intrinsic nonrandomness.
\end{abstract}

Subject headings: cosmology: observations - gamma rays: bursts

\section{INTRODUCTION}

Two recent results in the statistics of gamma-ray bursts (GRBs) are doubtlessly remarkable. The first one concerns the number of subclasses. Two different articles (Mukherjee et al. 1998; Horváth 1998) have simultaneously suggested that the earlier separation of GRBs into short and long subclasses (Kouveliotou et al. 1993) is incomplete. (It is a common practice to call GRBs having $T_{90}<2 \mathrm{~s}\left[T_{90}>2 \mathrm{~s}\right]$ "short" ["long"] GRBs, where $T_{90}$ is the time during which $90 \%$ of the fluence is accumulated [Kouveliotou et al. 1993].) These articles show that, in essence, the earlier "long" subclass should be further separated into a new, "intermediate" subclass $\left(2 \mathrm{~s}<T_{90}<10 \mathrm{~s}\right)$ and a "truncated long" subclass $\left(T_{90}>10 \mathrm{~s}\right)$. (In what follows, the "long" subclass will contain only the GRBs with $T_{90}>10$ $\mathrm{s}$, and the intermediate subclass will be considered as a new subclass.)

The second result concerns the angular distribution of GRBs. In recent years several attempts (Hartmann, Linder, \& Blumenthal 1991; Briggs et al. 1996; Tegmark et al. 1996b; Balázs, Mészáros, \& Horváth 1998; Balázs et al. 1999) were made to either confirm or reject the randomness in the angular sky distribution of GRBs being collected in the BATSE Catalog (Fishman et al. 1994; C. A. Meegan et al. 1998, Current BATSE Catalog 7 ). Theoretically, even if the intrinsic distribution of GRBs is actually random, an observation of some nonrandomness is still expected, due to the BATSE nonuniform sky-exposure function (Fishman et al. 1994; Meegan et al. 1998). Hartmann et al. (1991), Briggs et al. (1996), and Tegmark et al. (1996b) did not find any statistically significant departure from randomness. On the

\footnotetext{
${ }^{1}$ Department of Astronomy, Charles University, V Holešovičkách 2, CZ-180 00 Prague 8, Czech Republic.

${ }^{2}$ Konkoly Observatory, Box 67, H-1505 Budapest, Hungary.

${ }^{3}$ Department of Astronomy, Eötvös University, Pázmány Péter sétány 1/A, H-1518 Budapest, Hungary.

${ }^{4}$ Laboratory for Information Technology, Eötvös University, Pázmány Péter sétány 1/A, H-1518 Budapest, Hungary.

${ }^{5}$ Department of Physics, BJKMF, Box 12, H-1456 Budapest, Hungary.

${ }^{6}$ Observatoire de Paris, F-92195 Meudon Cedex, France.

${ }^{7}$ C. A. Meegan et al. 1998, Current BATSE Gamma-Ray Burst Catalog, http://cossc.gsfc.nasa.gov/batse.
}

other hand, the existence of some nonrandomness was confirmed on the greater than $99.9 \%$ confidence level by Balázs et al. (1998). Either this behavior can be caused purely by instrumental effects, or the instrumental effects alone do not explain fully the detected behavior and some intrinsic nonrandomness should also exist. Balázs et al. $(1998,1999)$ suggest the second possibility. This conclusion follows from the result that while the short subclass shows a nonrandomness, the intermediate and long subclasses do not. It is difficult to explain such behavior of subclasses by instrumental effects alone.

In this article we will again investigate the angular distribution of GRBs. After the discovery of the new, intermediate subclass, testing the intrinsic randomness in the angular distribution of this new subclass is highly required. In addition, of course, new, different tests, which exactly eliminate the effect of the sky-exposure function, are also required in order to complete the results of Balázs et al. $(1998,1999)$.

The aim of this article is to test the intrinsic randomness in the angular distribution of all GRBs together and of the three subclasses individually. We will use a modification of the well-known counts-in-cells method. This is a standard and simple statistical test (see, e.g., Mészáros 1997 and references therein). The advantage of this method is given by the fact that it allows the elimination, quite simply and exactly, of the sky-exposure function. The main result of this article will be the surprising conclusions that the intermediate subclass, and only this subclass, suggests a nonrandomness on the $96.4 \%$ confidence level and that its "dimmer" half suggests a nonrandomness on the even higher $99.3 \%$ confidence level.

The article is organized as follows. In $\S 2$ the method is described. In $\S 3$ the results of the test are presented. Section 4 discusses and summarizes the results.

\section{THE TEST}

Assume for the moment that there is no nonuniform skyexposure function. We separate the sky in declination, $\delta$, into $m_{\text {decl. }}>1$ stripes having the same area $\left(4 \pi / m_{\text {decl. }}\right.$. steradians). The boundaries of the stripes are the declinations $\delta_{k}, k=0,1, \ldots, m_{\text {decl. }}$, where $\delta_{0}=-90^{\circ}$ and $\delta_{m_{\text {decl. }}}=+90^{\circ}$, respectively. The remaining values are ana- 
lytically calculable and appear symmetrically with respect to $\delta=0$. One has $\sin \delta_{k}=2 k / m_{\text {decl. }}-1$. (For example, if $m_{\text {decl. }}=3$, then $\delta_{1,2}= \pm 19.47$; if $m_{\text {decl. }}=4$, then $\delta_{1,3}=$ \pm 30.00 and $\delta_{2}=0.00$; etc.) We also separate the sky in right ascension, $\alpha$, into $m_{\text {R.A. }}>1$ stripes. They are defined by boundaries $\alpha=360 k^{\prime} / m_{\text {R.A. }}{ }^{\circ} ; \quad k^{\prime}=0,1, \ldots, m_{\text {R.A. }}$. [Obviously, a trivial modification of this separation in right ascension is the case when the boundaries are $\alpha=360\left(k^{\prime}\right.$ $+p) / m_{\text {R.A. }}{ }^{\circ}$, where $p$ is an arbitrary real number fulfilling $0<p<1$.] All this means that we have separated the sky into $M=m_{\text {decl. }} \times m_{\text {R.A. }}$ areas ("cells") having the same size, $4 \pi / M$ steradians. If there are $N$ GRBs on the sky, then $n=N / M$ is the mean of GRBs in a cell. Let $n_{i}, i=$ $1,2, \ldots, M$, be the observed number of GRBs at the $i$ th cell $\left(\sum_{i=1}^{M} n_{i}=N\right)$. Then

$$
\operatorname{var}_{M}=(M-1)^{-1} \sum_{i=1}^{M}\left(n_{i}-n\right)^{2}
$$

defines the observed variance. For the given cell structure with $M$ cells, due to the Bernoulli distribution (Mészáros 1997; Balázs et al. 1998), the measured variance $\operatorname{var}_{M}$ should be identical to the theoretically expected value $n(1-1 / M)$. This theoretical prediction should then be tested.

Note that this and similar methods (see, e.g., Mészáros 1997 for details and further references) are usual in astronomy. For example, this method was used already by Abell (1958) to reject randomness in the sky distribution of clusters of galaxies. Compared to other statistical tests ("two-point angular correlation function," "nearest neighbor distances," etc.; see Peebles 1980; Diggle 1983; Pásztor 1993), this test is not the most sensitive one for detecting nonrandomness. Its importance for our purposes is given by the fact that it allows an extremely simple generalization to the case with a nonzero sky-exposure function.

Now we generalize the method to this case. This may easily be done by changing the boundaries of cells in order to have the same probability (and hence the same expected number $n=N / M$ ) for a given cell. The sky-exposure function is a function of declination only (Fishman et al. 1994; Meegan et al. 1998). Hence, the choice of equatorial coordinates is highly convenient, because then no changes of boundaries are necessary in right ascension. The new boundaries $\delta_{k}, k=0,1, \ldots, m_{\mathrm{decl} .}$, in declination may be calculated analytically as follows. Clearly, $\delta_{0, m_{\text {decl. }}}= \pm 90^{\circ}$ remains. In the BATSE Catalog (Meegan et al. 1998) the exposure function $f(\delta)$ is defined for 37 values of declination (for $\delta_{r}=-90^{\circ},-85^{\circ}, \ldots,+85^{\circ},+90^{\circ} ; r=0,1,2, \ldots, 36$ ). To obtain $\delta_{k}$, first we calculate the value

$$
A=\frac{5 \pi}{180} \sum_{r=1}^{35} f\left(\delta_{r}\right) \cos \delta_{r},
$$

where for the given $r$ the corresponding declination is $\delta_{r}=$ $(-90+5 r)^{\circ}$. Note that $r=0$ and $r=36$ need not be in the sum, because $\cos \left( \pm 90^{\circ}\right)=0$. Then, second, for $m_{\text {decl. }} \geq 2$ we search for the values $\delta_{k}, k=1,2, \ldots,\left(m_{\text {decl. }}-1\right)$, as follows. For the given $k$ we search for the declination $\delta_{i}$ fulfilling the condition

$$
\frac{\pi}{36 A} \sum_{j=1}^{i} f\left(\delta_{j}\right) \cos \delta_{j} \leq \frac{k}{m_{\mathrm{decl} .}}<\frac{\pi}{36 A} \sum_{j=1}^{i+1} f\left(\delta_{j}\right) \cos \delta_{j} .
$$

Having this, we search by linear interpolation between $\delta_{i}$ and $\delta_{i+1}$ for the exact value of $\delta_{k}$. By this method $\delta_{k}$ is easily calculable. (For example, for $m_{\text {decl. }}=3$ we obtain $\delta_{1}=-19.51, \delta_{2}=22.44$; for $m_{\text {decl. }}=4$ we obtain $\delta_{1}=$ $-30.83, \delta_{2}=1.51, \delta_{3}=33.60$; etc.) Having these cells with these "shifted" boundaries in declination, the variance may be calculated identically to the case with no sky-exposure function. This method will test the pure intrinsic randomness; the effect of the BATSE sky-exposure function is exactly eliminated.

It is natural to probe different values of $M$. In addition, for some $M$, different cell structures are still possible (e.g., $M=12$ allows $m_{\text {decl. }}=2,3,4,6$ ). Hence, generally, several - say $Q$ - cell structures may be probed for the same sample of GRBs. Having these $Q$ cell structures (and hence $Q$ means $+Q$ measured variances) two questions arise: (1) How are we to calculate the confidence level for a given cell structure? and (2) Having $Q$ confidence-level values, how are we to calculate the final confidence level? The answer to the first question seems to be quite clear: $\operatorname{var}_{M} / n$ seems to be identical to the $\chi^{2}$ value for $M-1$ degrees of freedom (Trumpler \& Weaver 1953; Kendall \& Stuart 1969; Press et al. 1992; the mean is obtained from the sample itself, and therefore the degrees of freedom is $M-1$ ). Nevertheless, the situation is not so obvious, because the $\chi^{2}$ test needs $n>5$ (Trumpler \& Weaver 1953; Kendall \& Stuart 1969; Press et al. 1992). In addition, some statistical textbooks propose to use "quadratic" cells only (Diggle 1983, p. 23). If all these restrictions were taken into account, then $\chi^{2}$ tests would be possible only for $2 m_{\text {decl. }}=m_{\text {R.A. }}, M=2 m_{\text {decl. }}^{2}$, and $N>5 M=10 m_{\text {decl. }}^{2}$. This would be a drastic truncation of the possible cell structures. But, not doing these restrictions, the estimation of the confidence level for a given cell structure must be done by more complicated procedures; e.g., by numerical simulations. Concerning the answer to the second question, the situation is even less clear. As a reasonable search for the final confidence level, only Monte Carlo simulations seem to be usable (Press et al. 1992).

Keeping all this in mind, we will proceed as follows. In the coordinate system with axes $x=1 / M$ versus $y=$ $\sqrt{\operatorname{var}_{M} / n}=(\operatorname{var} / \text { mean })^{1 / 2}$, the $Q$-values of $(\operatorname{var} / \text { mean })^{1 / 2}$ define $Q$ points (one point for any cell structure; $y_{j}=$ $\sqrt{\operatorname{var}_{M, j} / n}$, where $\left.j=1,2, \ldots, Q\right)$. Clearly, for these points one expects the theoretical curve $y=\sqrt{1-x}$. This theoretical expectation can straightforwardly be verified, e.g., by least-squares estimation (Press et al. 1992, p. 655; Diggle 1983 , p. 74). Our estimator is the dispersion

$$
\sigma_{Q}=\sum_{j=1}^{Q}\left(y_{j}-\sqrt{1-1 / M}\right)^{2} .
$$

Obviously, smaller $\sigma_{Q}$ suggests that the theoretical curve is better fitted. Note still that, as the best choice, the square root of $\operatorname{var}_{M} / n$ is proposed in this "var/mean" test (Diggle 1983, p. 77).

The confidence level can then be estimated by Monte Carlo simulations in the following way: We throw $N$ points on the sphere 1000 times, randomly, and repeat the above calculation leading to $\sigma_{Q}$ for every simulated sample. Then we compare the size of the $\sigma_{Q}$ obtained from this simulation with $\sigma_{Q}$ obtained from the actual GRB positions. Let $\omega$ be the number of simulations, when the obtained $\sigma_{Q}$ is greater than the actual value of $\sigma_{Q}$. Then one may conclude that $100-\omega / 10$ is the confidence level in percentage. Clearly, this method does not need $n>5$ and quadratic cells.

There is no commonly accepted confidence level in statistics above which the null hypothesis should already be 


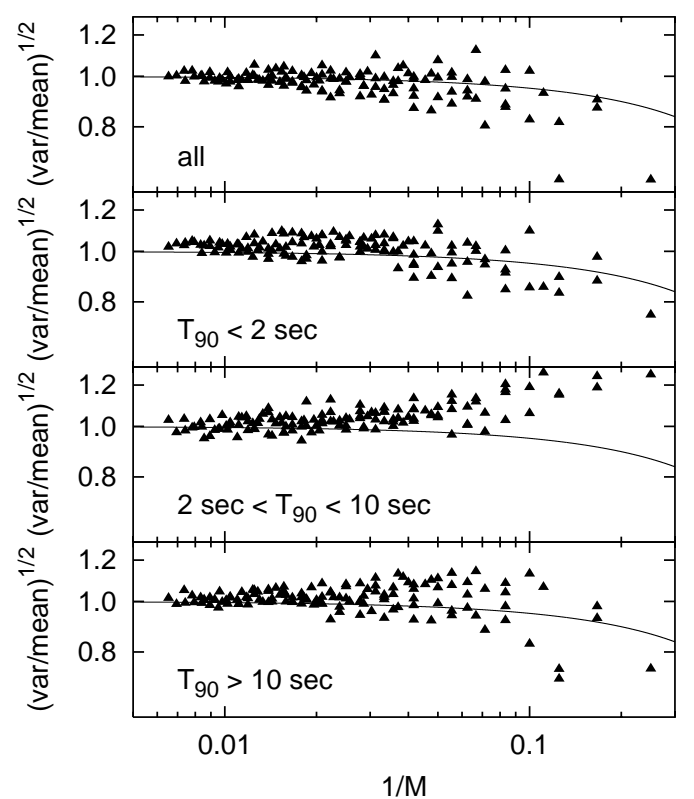

FIG. 1.-The results of 105 "var/mean" tests of four different cases drawn in the $1 / M$ vs. $\sqrt{\operatorname{var} / \text { mean }}$ frame. The theoretical curve $\sqrt{1-1 / M}$ (solid line) is also shown. $M$ is the number of cells.

rejected (Trumpler \& Weaver 1953; Kendall \& Stuart 1969). It is only a general agreement that confidence levels less than $95 \%$ should not be considered. Our opinion is that confidence levels greater than $95 \%$ can already be taken as "remarkable," "suspicious," "interesting," etc. (see also Kendall \& Stuart 1969); a greater than $99 \%$ confidence level may mean the rejection of the null hypothesis, and such result must doubtlessly be announced. Hence, we will require that the confidence level be greater than $95 \%$. Thus, here it must be $\omega<50$.

This article will use GRBs between trigger numbers 0105 and 6963 in the Current BATSE Catalog (Meegan et al. 1998) having defined $T_{90}$ (i.e., all GRBs detected up to 1998 August having measured $T_{90}$ ). From them we exclude, similarly to Pendleton et al. (1997) and Balázs et al. (1998), the faintest GRBs, i.e., those having a peak flux (on $256 \mathrm{~ms}$ trigger) of less than 0.65 photons $\mathrm{cm}^{-2} \mathrm{~s}^{-1}$. This truncation is proposed by Pendleton et al. (1997) in order to avoid the problems with the changing threshold. The 1284 GRBs obtained in this way define the "all" class. From this there are 339 GRBs with $T_{90}<2 \mathrm{~s}$ (the "short" subclass), 181 GRBs with $2 \mathrm{~s}<T_{90}<10 \mathrm{~s}$ (the "intermediate" subclass), and 764 GRBs with $T_{90}>10 \mathrm{~s}$ (the "long" subclass). We will study the all class and the three subclasses separately.

We will choose ad hoc $m_{\text {decl. }}=2,3, \ldots, 8$ and $m_{\text {R.A. }}=$ $2,3, \ldots, 16$. Thus, $Q=105$. Of course, this choice of $Q$ is more or less subjective. Nevertheless, our choice is motivated by two concrete arguments. First, we would like to study only the angular scales much greater than the positional errors. (The size of a cell will not be less than 22.5 . On these angular scales no problems should arise from the positional errors [Meegan et al. 1998].) Second, it is reasonable not to consider such high values of $m_{\text {decl. }}$, when $180 / m_{\text {decl. }}$ is already comparable to or even less than $5^{\circ}$. (If this were not required, then the elimination of the sky-exposure function would be problematic because of its definition for declination intervals with widths of $5^{\circ}$.)

\section{THE RESULTS}

Figure 1 collects the results of the $Q=105$ "var/mean" tests of four different cases. It is obvious immediately that for the "all" case the points follow well the theoretical curve. For the short and long subclasses, on the other hand, there is a slight tendency of points to be above the theoretical curve. The situation concerning the intermediate subclass seems to be the most unambiguous: mainly for small $M$ (roughly below $M \simeq 40$ ) the points are clearly above the theoretical curve. This suggests an intrinsic nonrandomness in the sky distribution mainly of the intermediate subclass; but such a possibility cannot be excluded for the short and long subclasses.

The results of the Monte Carlo simulations support this expectation only in the case of the intermediate subclass. We obtain $\omega=287, \omega=80, \omega=36$, and $\omega=440$ for all, short, intermediate, and long GRBs, respectively. Hence, the rejection of the null hypothesis is confirmed for the intermediate subclass only, on the $96.4 \%$ confidence level. For the short and long subclasses, and also for all GRBs together, the null hypothesis cannot be rejected on the greater than $95 \%$ confidence level. For the short subclass we have a $92 \%$ confidence level; for the remaining two cases, even lower levels.

\section{DISCUSSION AND CONCLUSION}

The most surprising result of this article concerns the intermediate subclass, the intrinsic nonrandomness of which is confirmed on the greater than $95 \%$ confidence level. This confidence level, as discussed in $\S 2$, is "remarkable" but is not high enough to reject the null hypothesis of randomness.

The results concerning the 339 short GRBs should also be mentioned. The $92 \%$ confidence level is clearly not high enough to reject the null hypothesis. On the other hand, this result, together with those of Balázs et al. $(1998,1999)$, suggests that the rejection of the null hypothesis of intrinsic randomness may occur for the short subclass also, through further tests.

In the case of the 764 long GRBs, and also of all 1284 GRBs together, there are no indications of nonrandomness. All this seems to be in accordance with the results of Balázs et al. $(1998,1999)$.

We think that the result concerning the intermediate subclass is highly surprising, because only this new subclass, having the smallest number of GRBs, has a remarkable "proper" behavior. A short further investigation of this subclass fully supports this conclusion.

There are 181 GRBs in this intermediate subclass. This subclass can be divided into two further subclasses: the "dim" and "bright" ones. By chance the median peak flux for the intermediate subclass is almost exactly 2 photons $\mathrm{cm}^{-2} \mathrm{~s}^{-1}$ (on $0.256 \mathrm{~s}$ trigger). Therefore, we consider the GRBs having peak fluxes of less than (greater than) 2 photons $\mathrm{cm}^{-2} \mathrm{~s}^{-1}$ as the "dim" ("bright") subclass of the intermediate subclass. There are 92 GRBs in the dim subclass and 89 GRBs in the bright one.

We perform the 105 "var/mean" tests for each of these subclasses also. We obtain the surprising result that the dim subclass has an intrinsic nonrandomness on the $99.3 \%$ confidence level $(\omega=7)$. Contrary to this, the bright subclass is random $(\omega=662)$. The sky distribution of the 92 dim intermediate GRBs is shown in Figure 2. 


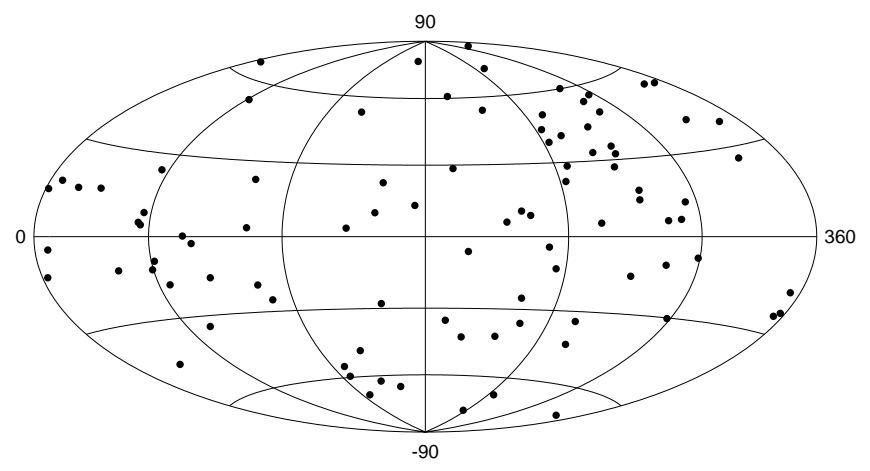

FIG. 2.-Sky distribution of the 92 GRBs of the dim subclass of the intermediate subclass in equatorial coordinates.

We believe that the behavior of the intermediate subclass of GRBs, quite independently, supports the correctness of the introduction of this new subclass by Mukherjee et al. (1998) and by Horváth (1998). Further investigations of this new subclass are highly required.

Three notes are still needed. First, purely from the statistical point of view, we must be precise in saying that even the rejection of the null hypothesis of intrinsic randomness would not mean a pure intrinsic nonrandomness in the spatial angular distribution of GRBs. This is given by the fact that, at present, it cannot be fully excluded that not all GRBs are unique and that some of them are repeating. This question is studied intensively in several papers (Meegan et al. 1995; Quashnock 1996a, 1996b; Tegmark et al. 1996a; Graziani, Lamb, \& Quashnock 1998; Hakkila et al. 1998) which conclude that repetition can still play a role.

Second, strictly speaking, the statistical counts-in-cells test is testing the "complete spatial randomness" (or simply the "randomness") of the distribution of GRBs on the celestial sphere (Diggle 1983, p. 4). Therefore, in this article we have kept this terminology. In cosmology, on the other hand, the word "random" ("nonrandom") is rarely used, and the word "isotropic" ("anisotropic") is usual (for the exact definition of "isotropy" in cosmology see, e.g., Weinberg 1972). Of course, here we will not go into the details of these terminology questions (see, e.g., Peebles 1980 for more details concerning these questions). We note only that the "random-isotropic" ("nonrandom-anisotropic") substitution is quite acceptable on the greatest angular scales; on lesser angular scales the situation is not so clear. Therefore, in Balázs et al. (1998, 1999), where only the angular scales of $\sim 90^{\circ}$ and higher were studied, the words "isotropy" and "anisotropy" were quite usable. In this article, going down to scales of about $20^{\circ}-25^{\circ}$, the question of terminology is more relevant.

Third, at the very least, further studies are needed. They should test again - by different statistical methods - the intrinsic randomness (more generally, the intrinsic spatial distribution; see Lamb 1997), of all GRBs together and of the individual subclasses. In addition, a test of repetition alone, i.e., a test not influenced by positions, is highly required.

In conclusion, the results of this article can be summarized as follows:

1. We developed a method which can verify quite simply the intrinsic randomness in the angular distribution of GRBs by eliminating exactly the nonzero sky-exposure function.

2. We rejected the null hypothesis of intrinsic randomness in the angular distribution of 181 intermediate GRBs on the $96.4 \%$ confidence level.

3 . We rejected the null hypothesis of intrinsic randomness in the angular distribution of 92 dim intermediate GRBs on the $99.3 \%$ confidence level.

4. We did not reject the null hypotheses of intrinsic randomness in the angular distribution of the remaining two subclasses and of all GRBs together on the greater than 95\% confidence level; the bright intermediate GRBs seem to be randomly distributed also.

We thank Michael Briggs, Peter Mészáros, László Pásztor, Dennis Sciama, and Gábor Tusnády for valuable discussion, and we thank the anonymous referee. A. M. thanks Konkoly Observatory and Eötvös University for their hospitality. This article was partly supported by GAUK grant 36/97, by GAČR grant 202/98/0522, by a Domus Hungarica Scientiarum et Artium grant (A. M.), by OTKA grant T024027 (L. G. B.), and by OTKA grant F029461 (I. H.).

\section{REFERENCES}

Abell, G. O. 1958, ApJS, 3, 211

Balázs, L. G., Mészáros, A., \& Horváth, I. 1998, A\&A, 339, 1

Balázs, L. G., Mészáros, A., Horváth, I., \& Vavrek, R. 1999, A\&AS, 138, 417

Briggs, M. S., et al. 1996, ApJ, 459, 40

Diggle, P. J. 1983, Statistical Analysis of Spatial Point Patterns (London: Academic)

Fishman, G. J., et al. 1994, ApJS, 92, 229

Graziani, C., Lamb, D. Q., \& Quashnock, J. M. 1998, in AIP Conf. Proc. 428, 4th Huntsville Symp. on Gamma-Ray Bursts, ed. C. A. Meegan, R. D. Preece, \& T. M. Koshut (New York: AIP), 161

Hakkila, J., Meegan, C. A., Pendleton, G. N., Briggs, M. S., Horack, J. M., Hartmann, D. H., \& Connaughton, V. 1998, in AIP Conf. Proc. 428, 4th Huntsville Symp. on Gamma-Ray Bursts, ed. C. A. Meegan, R. D. Preece, \& T. M. Koshut (New York: AIP), 236

Hartmann, D. H., Linder, E. V., \& Blumenthal, G. R. 1991, ApJ, 367, 186

Horváth, I. 1998, ApJ, 508, 757

Kendall, M. G., \& Stuart, A. 1969, Advanced Theory of Statistics, Vol. 1 (London: Griffin)

Kouveliotou, C., Meegan, C. A., Fishman, G. J., Bhat, N. P., Briggs, M. S., Koshut, T. M., Paciesas, W. S., \& Pendleton, G. N. 1993, ApJ, 413, L101 Lamb, D. Q. 1997, Rev. Mod. Astron., 10, 101
Meegan, C. A., et al. 1995, ApJ, 446, L15

Mészáros, A. 1997, A\&A, 328, 1

Mukherjee, S., Feigelson, E. D., Babu, G. J., Murtagh, F., Fraley, C., \& Raftery, A. 1998, ApJ, 508, 314

Pásztor, L. 1993, Ph.D. thesis, Eötvös Univ.

Peebles, P. J. E. 1980, The Large-Scale Structure of the Universe (Princeton: Princeton Univ. Press)

Pendleton, C. N., et al. 1997, ApJ, 489, 175

Press, W. H., Teukolsky, S. A., Vetterling, W. T., \& Flannery, B. P. 1992, Numerical Recipes in FORTRAN (2d ed.; Cambridge: Cambridge Univ. Press)

Quashnock, J. M. 1996a, in AIP Conf. Proc. 366, High Velocity Neutron Stars and Gamma-Ray Bursts, ed. R. E. Rothschild \& R. E. Lingenfelter (Woodbury, N.Y.: AIP)

.1996b, ApJ, 461, L69

Tegmark, M., Hartmann, D. H., Briggs, M. S., Hakkila, J., \& Meegan, C. A. 1996a, ApJ, 466, 757

Tegmark, M., Hartmann, D. H., Briggs, M. S., \& Meegan, C. A. 1996b, ApJ, 468, 214

Trumpler, R. J., \& Weaver, H. F. 1953, Statistical Astronomy (Berkeley: Univ. California Press)

Weinberg, S. 1972, Gravitation and Cosmology (New York: Wiley) 